\title{
Characterization of Oligomeric Species on the Aggregation Pathway of Human Lysozyme
}

\author{
Erica Frare ${ }^{1} \dagger$, Maria F. Mossuto ${ }^{1,2} \dagger$, Patrizia Polverino de Laureto ${ }^{1}$, \\ Serena Tolin ${ }^{1}$, Linda Menzer ${ }^{2}$, Mireille Dumoulin ${ }^{2}$, \\ Christopher M. Dobson ${ }^{2}$ and Angelo Fontana ${ }^{1 *}$
}

${ }^{1}$ CRIBI Biotechnology Centre, University of Padua, Viale G. Colombo 3, 35121 Padua, Italy

${ }^{2}$ Department of Chemistry, University of Cambridge, Lensfield Road, Cambridge CB2 1EW, UK

Received 9 October 2008; received in revised form 23 December 2008; accepted 25 January 2009 Available online 30 January 2009

\begin{abstract}
The aggregation process of wild-type human lysozyme at $\mathrm{pH} 3.0$ and $60^{\circ} \mathrm{C}$ has been analyzed by characterizing a series of distinct species formed on the aggregation pathway, specifically the amyloidogenic monomeric precursor protein, the oligomeric soluble prefibrillar aggregates, and the mature fibrils. Particular attention has been focused on the analysis of the structural properties of the oligomeric species, since recent studies have shown that the oligomers formed by lysozyme prior to the appearance of mature amyloid fibrils are toxic to cells. Here, soluble oligomers of human lysozyme have been analyzed by a range of techniques including binding to fluorescent probes such as thioflavin $\mathrm{T}$ and 1-anilino-naphthalene-8-sulfonate, Fourier transform infrared spectroscopy, and controlled proteolysis. Oligomers were isolated after 5 days of incubation of the protein and appear as spherical particles with a diameter of 8-17 $\mathrm{nm}$ when observed by transmission electron microscopy. Unlike the monomeric protein, oligomers have solventexposed hydrophobic patches able to bind the fluorescent probe 1-anilinonaphthalene-8-sulfonate. Fourier transform infrared spectroscopy spectra of oligomers are indicative of misfolded species when compared to monomeric lysozyme, with a prevalence of random structure but with significant elements of the $\beta$-sheet structure that is characteristic of the mature fibrils. Moreover, the oligomeric lysozyme aggregates were found to be more susceptible to proteolysis with pepsin than both the monomeric protein and the mature fibrils, indicating further their less organized structure. In summary, this study shows that the soluble lysozyme oligomers are locally unfolded species that are present at low concentration during the initial phases of aggregation. The nonnative conformational features of the lysozyme molecules of which they are composed are likely to be the factors that confer on them the ability to interact inappropriately with a variety of cellular components including membranes.
\end{abstract}

(C) 2009 Elsevier Ltd. All rights reserved.
${ }^{*}$ Corresponding author. E-mail address:

angelo.fontana@unipd.it.

Present addresses: M. F. Mossuto, Institute for Research in Biomedicine, Baldiri Reixac 10, 08028 Barcelona, Spain; M. Dumoulin, Centre for Protein Engineering, Institute of Chemistry B6, University of Liège, B4000 Liège, Belgium.

$\dagger$ E.F. and M.F.M. contributed equally to this work.

Abbreviations used: ANS, 1-anilino-naphthalene-8-sulfonate; FTIR, Fourier transform infrared spectroscopy; TEM, transmission electron microscopy; ThT, thioflavin $\mathrm{T}$.

\section{Introduction}

Several lines of evidence have recently suggested that prefibrillar protein aggregates formed prior to the appearance of mature fibrils are likely to represent the most toxic entities responsible for cell dysfunction and death in at least some forms of amyloid disease. ${ }^{1,2}$ In particular, nonfibrillar aggregates from several amyloid-forming pathological and nonpathological proteins have been shown to be 
toxic to cultured fibroblasts and pheochromocytoma cells. ${ }^{3,4}$ The pathological importance of the soluble amyloid oligomers is, however, very challenging to assess since they are difficult to extract from the brain and other types of tissue. Moreover, a detailed analysis of the structure of the oligomers at the molecular level, and of the mechanism of their formation, is difficult because these species are often unstable and can exist in dynamic equilibrium with monomers, oligomers of different sizes, and higher molecular weight aggregates including fibrils. ${ }^{5}$

A form of systemic nonneuropathic amyloidosis is associated with the deposition in various organs of amyloid fibrils formed by single point mutational variants of human lysozyme, namely, I56T, F57I, W64R, and $\mathrm{D} 67 \mathrm{H}^{6-8}$ and two variants with double mutations F57I/T70N and W112R/T70N. ${ }^{8,9}$ Recently, evidence that prefibrillar aggregates formed in vitro from wild-type hen and horse lysozyme are toxic to cell cultures has emerged, causing an increase in membrane permeability and a disruption in the regulation of $\mathrm{Ca}^{2+}$ ions ${ }^{10}$ and, ultimately, cell death in a range of cell lines. ${ }^{11,12}$ Moreover, injection of such oligomeric species into rat brains leads to neurodegeneration, whereas mature amyloid fibrils are less damaging or nontoxic. ${ }^{13}$

In the present study, the aggregation process of wild-type human lysozyme has been investigated, with the particular aim of providing details of the structure of the species formed early during the aggregation process. In a previous study, it was shown by a combined approach of limited proteolysis and Fourier transform infrared spectroscopy (FTIR) that the core region of mature fibrils from human lysozyme produced in vitro at low $\mathrm{pH}$ is primarily formed by residues $32-108$ in the 130-polypeptide chain of the protein and that the rest of the chain is relatively unstructured. ${ }^{14}$ The segment 32-108 corresponds to the region found to undergo cooperative local unfolding in the amyloidogenic variants I56T and D67H of human lysozyme ${ }^{15,16}$ and to be amyloidogenic in the homologous hen lysozyme. ${ }^{17,18}$ Given the high stability of wild-type human lysozyme, ${ }^{19-21}$ conditions such as low $\mathrm{pH}$ and high temperature or ethanol have been previously used to populate a partially unfolded state that is prone to form fibrils. ${ }^{14,22,23}$ However, these conditions known so far for the efficient formation of amyloid fibrils by human lysozyme were found not to be appropriate for this work because they either induce the fragmentation of the protein ${ }^{14,18}$ or lead to an aggregation process that is too fast to allow isolation of oligomeric species. ${ }^{14}$

In this work, soluble spherical lysozyme oligomers were identified and then isolated by ultrafiltration during the long lag phase of aggregation under partially denaturing but relatively mild conditions ( $\left.\mathrm{pH} 3.0,60{ }^{\circ} \mathrm{C}\right)$. The conformational features of these species have then been investigated by various techniques including transmission electron microscopy (TEM), binding to the fluorescent probes thioflavin $\mathrm{T}$ (ThT) and 1-anilino-naphthalene-8-sulfonate (ANS), FTIR, and controlled proteolysis. The results show that prefibrillar lysozyme aggregates have distinctive features when compared with both the monomeric protein and the mature fibrils. In particular, the FTIR data show that the $\alpha / \beta$-structure characteristic of native lysozyme undergoes partial unfolding on conversion into the oligomers, although a significant degree of $\beta$ structure forms and stabilizes the protein aggregates. Moreover, complete digestion of the lysozyme molecules present within the oligomers indicates that the polypeptide chain of lysozyme in the oligomers is locally more unfolded compared to both the monomeric protein and the final fibrils.

We suggest that the misfolded structure and the marked hydrophobic character of the oligomers deduced by ANS experiments can lead to a gain of toxicity following aggregation of an otherwise innocuous protein, as it indicates that the surface of these soluble aggregates is likely to have a high propensity to interact inappropriately with cellular components, notably membranes, and, hence, cause cellular damage and, ultimately, cell death. ${ }^{1,3}$

\section{Isolation of prefibrillar aggregates of human lysozyme}

The ability of human lysozyme to aggregate into amyloid fibrils generally increases under conditions that favor the formation of partially unfolded species. ${ }^{24,25}$ In order to identify suitable conditions for populating oligomeric species in significant amounts and on a time scale allowing their detailed characterization, we monitored the thermal unfolding of lysozyme at $\mathrm{pH} 3.0$ by far-UV circular dichroism (CD) and ANS fluorescence measurements (Fig. 1). A plot of the apparent fraction of folded protein as a function of temperature increase was derived from the values of ellipticity at $222 \mathrm{~nm}$ recorded during thermal denaturation experiments (Fig. 1). At pH3.0, the thermal unfolding follows an apparent two-state process, as already reported, ${ }^{19}$ with a temperature of midtransition $\left(T_{\mathrm{m}}\right)$ of $79^{\circ} \mathrm{C}$. The unfolding process of lysozyme was also followed by monitoring the fluorescence emission at $475 \mathrm{~nm}$ of ANS, a dye whose wavelength of maximum emission shifts from 514 to $475 \mathrm{~nm}$ upon binding to exposed hydrophobic patches. ${ }^{29}$ Interestingly, the ANS emission intensity at $475 \mathrm{~nm}$ starts increasing at $50{ }^{\circ} \mathrm{C}$ and reaches its maximum value at $76{ }^{\circ} \mathrm{C}$, after which it rapidly decreases (Fig. 1). This result reveals that partially unfolded species are populated in a range of temperature from 50 to $95{ }^{\circ} \mathrm{C}$, with a maximum population in close proximity to the temperature of midtransition determined by far-UV CD.

On the basis of these results, the experiments designed to probe the aggregation of lysozyme were conducted at $\mathrm{pH} 3.0$ and $60{ }^{\circ} \mathrm{C}$. Under these conditions, an ensemble of partially unfolded species able to bind ANS is significantly populated, corresponding approximately to $22 \%$ of the population present at the temperature of midtransition. Using higher temperature values for the aggregation experiments was avoided because even though it would have allowed the formation of a higher proportion of 
partially unfolded lysozyme molecules, it would also have increased the extent of acid-catalyzed hydrolysis of the polypeptide chain, mainly at Asp-X peptide

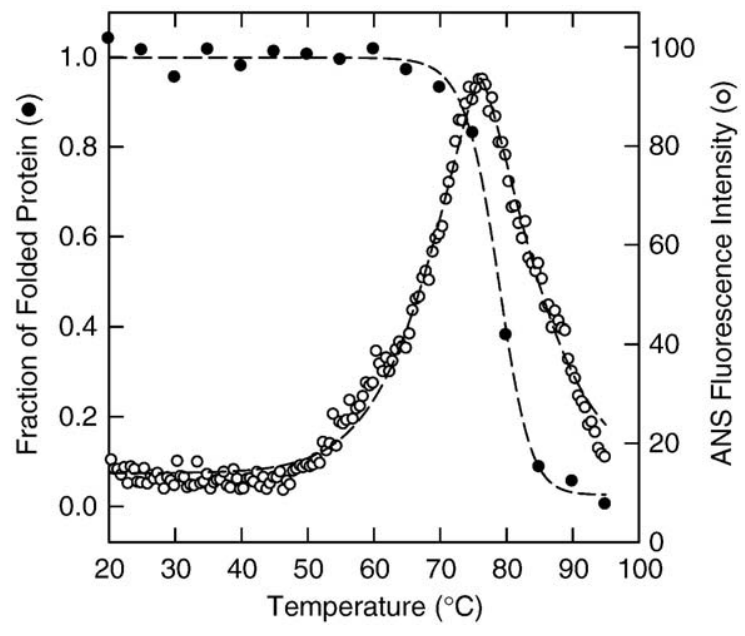

Fig. 1. Thermal denaturation curves of wild-type human lysozyme at pH3.0 followed by far-UV CD and ANS fluorescence. Filled and open circles represent, respectively, the apparent fraction of folded protein obtained from the ellipticity value at $222 \mathrm{~nm}$ and the fluorescence emission at $475 \mathrm{~nm}$ of ANS during thermal denaturation $\left(20-95^{\circ} \mathrm{C}\right)$ of human lysozyme in $20 \mathrm{mM}$ glycine $\cdot \mathrm{HCl}, \mathrm{pH} 3.0$. The apparent fraction of folded protein $f_{\text {app }}$ was calculated from the ellipticity at $222 \mathrm{~nm}$ versus temperature curve using the equation $f_{\text {app }}=\left([\theta]_{U}-\right.$ $[\theta]) /\left([\theta]_{U}-[\theta]_{N}\right)$, where $[\theta]$ represents the observed mean residue ellipticity under the given conditions and $[\theta]_{N}$ and $[\theta]_{\mathrm{U}}$ are the corresponding ellipticity values in the native $(\mathrm{N})$ and unfolded (U) states, respectively. The mean residue ellipticity $[\theta]\left(\mathrm{deg} \cdot \mathrm{cm}^{2} \cdot \mathrm{dmol}^{-1}\right)$ was calculated using the formula $[\theta]=\left(\theta_{\mathrm{obs}} / 10\right) \cdot(\mathrm{MRW} / \mathrm{lc})$, where $\theta_{\mathrm{obs}}$ is the observed ellipticity in degrees, MRW is the mean residue molecular weight, $l$ is the path length in centimeters, and $c$ is the protein concentration in grams per milliliter. The $[\theta]_{N}$ and $[\theta]_{U}$ values in the transition region were obtained by linear extrapolation of the ellipticity values observed in the temperature regions before and after the transition. Far-UV CD spectra were obtained with a Jasco J-710 (Tokyo, Japan) dichrograph, equipped with a thermostated cell holder. The instrument was calibrated with $d-(+) 10$-camphorsulfonic acid. Temperature-controlled cells of 1-mm path length and a protein concentration of $7 \mu \mathrm{M}$ were used for the far-UV CD measurements. ANS fluorescence emission was measured for $72-\mu \mathrm{M}$ solutions of ANS in the presence of a $2.4-\mu \mathrm{M}$ concentration of protein in $20 \mathrm{mM}$ glycine $\cdot \mathrm{HCl}$, $\mathrm{pH} 3.0$. The excitation wavelength was fixed at $350 \mathrm{~nm}$ and the fluorescence emission was collected at $475 \mathrm{~nm}$ using excitation and emission slits of $5 \mathrm{~nm}$, and the fluorescence signal of ANS in the absence of protein was subtracted from the one recorded in the presence of protein. Fluorescence emission measurements were conducted with a Perkin Elmer model LS-50B spectrofluorimeter (Norwalk, CT, USA), utilizing a $2-\mathrm{mm} \times 10-\mathrm{mm}$ path length cuvette. Protein concentrations were determined by absorption measurements at $280 \mathrm{~nm}$ on a double-beam Lambda-20 spectrophotometer from Perkin Elmer. The extinction coefficient $(\varepsilon)$ at $280 \mathrm{~nm}$ for lysozyme, evaluated on the basis of its amino acid composition, ${ }^{26,27}$ was $36,940 \mathrm{~cm}^{-1} \mathrm{M}^{-1}$, whereas the $\varepsilon$ at $350 \mathrm{~nm}$ of ANS was $4950 \mathrm{~cm}^{-1} \mathrm{M}^{-1} \cdot 28$ bonds. ${ }^{18,30}$ At pH3.0 and $60{ }^{\circ} \mathrm{C}$, moreover, the aggregation process is sufficiently slow to allow the isolation of distinct species along the pathway. In particular, after 5 days of incubation of the protein under the conditions described, TEM images show the presence of a large number of spherical protein aggregates with diameters ranging from 8 to $17 \mathrm{~nm}$ (Fig. 2, top). The morphology and size are in accord with the appearance of prefibrillar aggregates reported for hen lysozyme ${ }^{12,13}$ and indeed for other proteins. ${ }^{32-34}$ The approximate number of lysozyme molecules in each spherical aggregate can be estimated by counting the number of spheres with the diameter of the protein $(4.5 \mathrm{~nm})$ that can be fitted into spheres of the size of those observed by TEM, having diameters between 8 and $17 \mathrm{~nm}^{35}$ Following this approximation, lysozyme soluble oligomers would be constituted by $\sim 5-40$ protein monomers and would have a molecular mass in the range of 74-590 kDa. This number is similar to that defined in recent studies of other fibrils such as those formed by the $\mathrm{SH} 3$ domain of phosphoinositol-3-kinase. ${ }^{36}$ After 9 days of incubation, the oligomers are still present in the solution, as assessed by TEM: however, a number of them can be seen to be interacting with each other to form elongated structures. After 15 days of incubation, mature amyloid-like fibrils with a 9- to 14-nm diameter are evident in the micrographs (Fig. 2, top). These findings are consistent with a situation in which, during the course of the incubation process, the initially formed oligomers physically associate with each other, giving rise to filamentous structures that subsequently convert into mature fibrils.

In order to provide structural details about the early aggregates of lysozyme, we devised a method that was able to separate them from the monomeric protein. FTIR and CD spectra of nonpurified oligomer-containing samples (i.e., samples incubated under the conditions used here for 5 days) are nearly identical with those of the monomeric protein before incubation, where no aggregates are visible by TEM. This finding is probably due to the low concentration of soluble aggregates in the solution even after 5 days of incubation, such that the signals arising from the monomeric protein essentially overwhelm those coming from the oligomers. Ultracentrifugation of the solution at $380,000 \mathrm{~g}$ did not lead to the formation of any pellet, however, indicating that the lysozyme oligomers are highly soluble; indeed, the definition of soluble amyloid oligomers is based on their continued presence in the supernatant after very high speed centrifugation $(>370,000 \mathrm{~g}) \cdot{ }^{37,38}$ By contrast, mature fibrils are spun down after centrifugation at $<100,000 g^{39}$ thus, the ultracentrifugation of a lysozyme sample incubated at $\mathrm{pH} 3.0$ and $60{ }^{\circ} \mathrm{C}$ for 9 days, in which both oligomers and fibrils are present as indicated by TEM, leads to the partitioning of spherical aggregates in the supernatant and of fibrils in the pellet.

In the light of these results, lysozyme oligomers were isolated by ultrafiltration of samples after 5 days of incubation, which do not contain fibrils, 

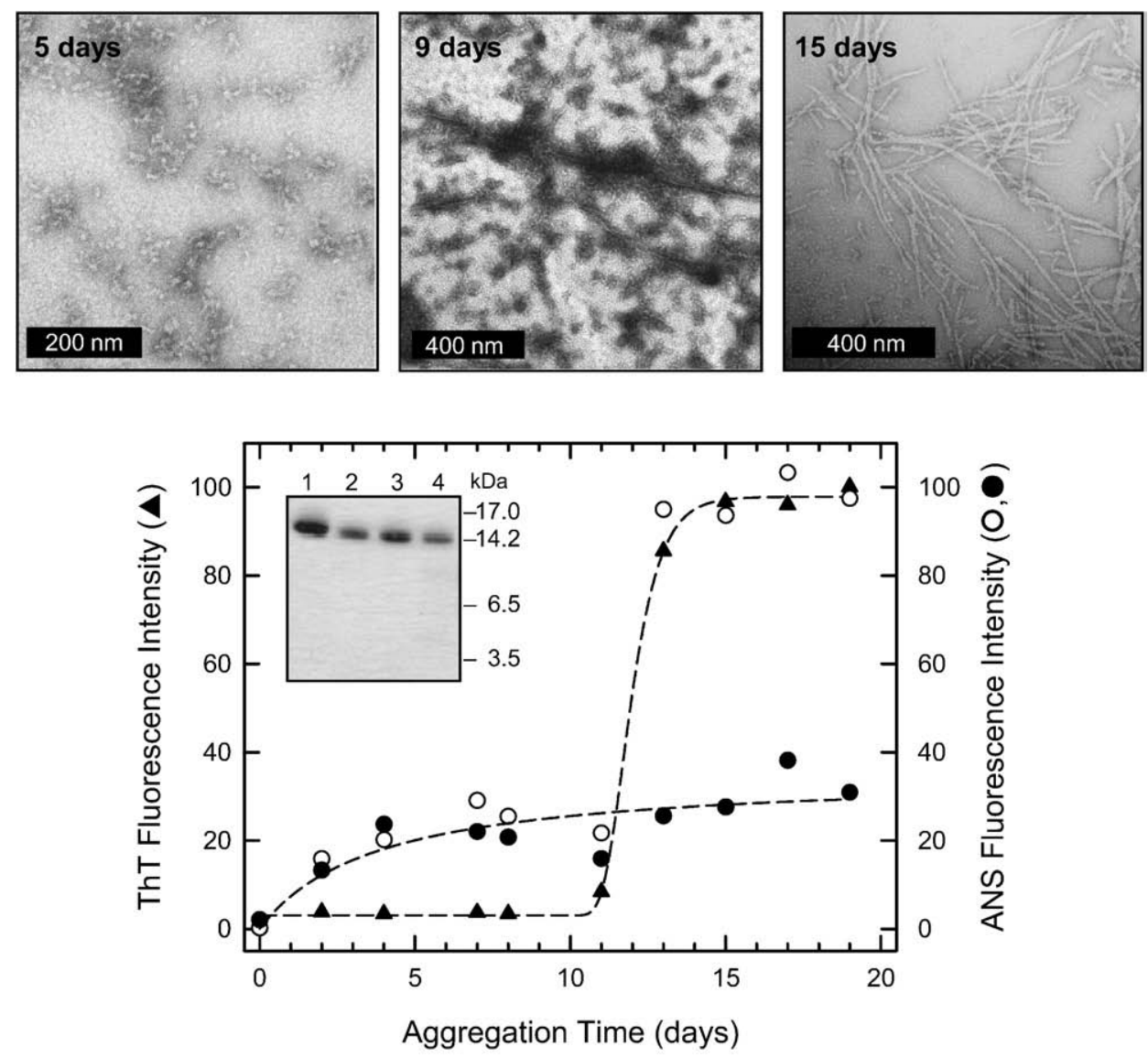

Fig. 2. Aggregation of human lysozyme at $\mathrm{pH} 3.0$ and $60^{\circ} \mathrm{C}$ monitored by (top) TEM and (bottom) ThT and ANS binding assays. Lysozyme was dissolved in $20 \mathrm{mM}$ glycine $\cdot \mathrm{HCl}, \mathrm{pH} 3.0$, at a $0.65 \mathrm{mM}(9 \mathrm{mg} / \mathrm{ml})$ concentration in order to induce the formation of protein aggregates. The solution was filtered with a 20-nm pore-size filter (Whatman, Maidstone, UK) and then incubated at $60^{\circ} \mathrm{C}$. TEM pictures were taken on a Tecnai $\mathrm{G}^{2} 12$ Twin instrument (FEI Company, Hillsboro, OR, USA), operating at an excitation voltage of $100 \mathrm{kV}$. Samples for TEM were diluted 10 times, and a drop of the solution was placed on a Butvar-coated copper grid (400-square mesh) (TAAB Laboratories Equipment Ltd., Berks, UK), followed by a drop of uranyl acetate solution (1\%, w/v). For the ThT assay, aliquots $(5 \mu \mathrm{l})$ of the protein solution, incubated for the periods of time indicated, were added to $25-\mathrm{mM}$ solutions $(496 \mu \mathrm{l})$ of ThT in $25 \mathrm{mM}$ phosphate buffer (pH6.0). The excitation wavelength was fixed at $440 \mathrm{~nm}$, and the fluorescence emission was collected at $485 \mathrm{~nm}$ (triangles). The maximum fluorescence value of ThT has been normalized to 100. For the ANS assay, aliquots from the solution in which aggregation was taking place were diluted with $20 \mathrm{mM}$ glycine· $\mathrm{HCl}, \mathrm{pH} 3.0$, and ANS was added to a molar ratio of protein:ANS of 1:2. The excitation wavelength was fixed at $350 \mathrm{~nm}$ and the fluorescence emission was collected at $475 \mathrm{~nm}$ at room temperature (circles). The maximum fluorescence intensity values of ANS have been normalized to 100. Open circles represent the fluorescence emission of ANS in the presence of the various aliquots, and filled circles represent that of the supernatants of the same aliquots obtained after ultracentrifugation for $90 \mathrm{~min}$ at $4{ }^{\circ} \mathrm{C}$ at $90,000 \mathrm{rpm}(380,000 \mathrm{~g})$ in a Beckman-Coulter MLA-130 ultracentrifuge (model Optima MAX-E, Palo Alto, CA). (Bottom, inset) SDS-PAGE analysis of standard human lysozyme (lane 1), of a 15-day aggregated lysozyme sample (lane 2), of the supernatant (lane 3), and of the pellet (lane 4) obtained after ultracentrifugation of a similar aggregated sample. SDS-PAGE was performed on $4-12 \%$ precast Bis-Tris NuPAGE gels (Invitrogen, Carlsbad, CA, USA) in 4-morpholineethanesulfonic acid buffer, under reducing conditions. Before SDSPAGE analysis, samples containing fibrils were dissolved in $95 \%$ dimethyl sulfoxide. ${ }^{31}$ The same amount of protein $(5 \mu \mathrm{g})$ was loaded in each well. A molecular weight standard was loaded onto the gel and the position of the relative bands is reported on the right.

through 100-kDa cutoff ultrafilters. The amount of lysozyme in the retentate was $\sim 5 \%$ of the total starting material, as estimated by UV absorption measurements, confirming that lysozyme oligomers are not highly populated species. In these samples, the material collected on a $100-\mathrm{kDa}$ filter and observed by TEM was composed of spherical species with an 8- to 17-nm diameter, similar to those observed in samples prior to centrifugation or ultrafiltration, indicating that the purification process does not affect the nature of the oligomers.

\section{Characterization of lysozyme oligomers}

The structural features of soluble lysozyme aggregates have been investigated by a range of techniques, including ANS and ThT binding fluorescence, FTIR, and proteolysis experiments. 
The fluorescence assay with ThT is indicative of the presence of ordered $\beta$-sheet structure in amyloid aggregates, ${ }^{40}$ and the kinetics of lysozyme fibril formation were monitored by following the increase of ThT fluorescence emission intensity during the aggregation process (Fig. 2, bottom, triangles). The experimental data points fit well to a sigmoidal curve, indicating the presence of an initial lag phase of about 10 days, followed by a rapid growth phase that reaches a plateau at 15 days. TEM pictures show that after 15 days of incubation, fibrils with typical amyloid morphology are present in the sample (Fig. 2 , top). These fibrils were separated from nonaggregated soluble material by ultracentrifugation; ca $90 \%$ of the total lysozyme content present in the initial solution was found in the pellet, as assessed by UV absorption measurements, indicating that almost all protein molecules had aggregated into fibrils. SDS-PAGE analysis of a 15-day sample was then performed in order to check the chemical

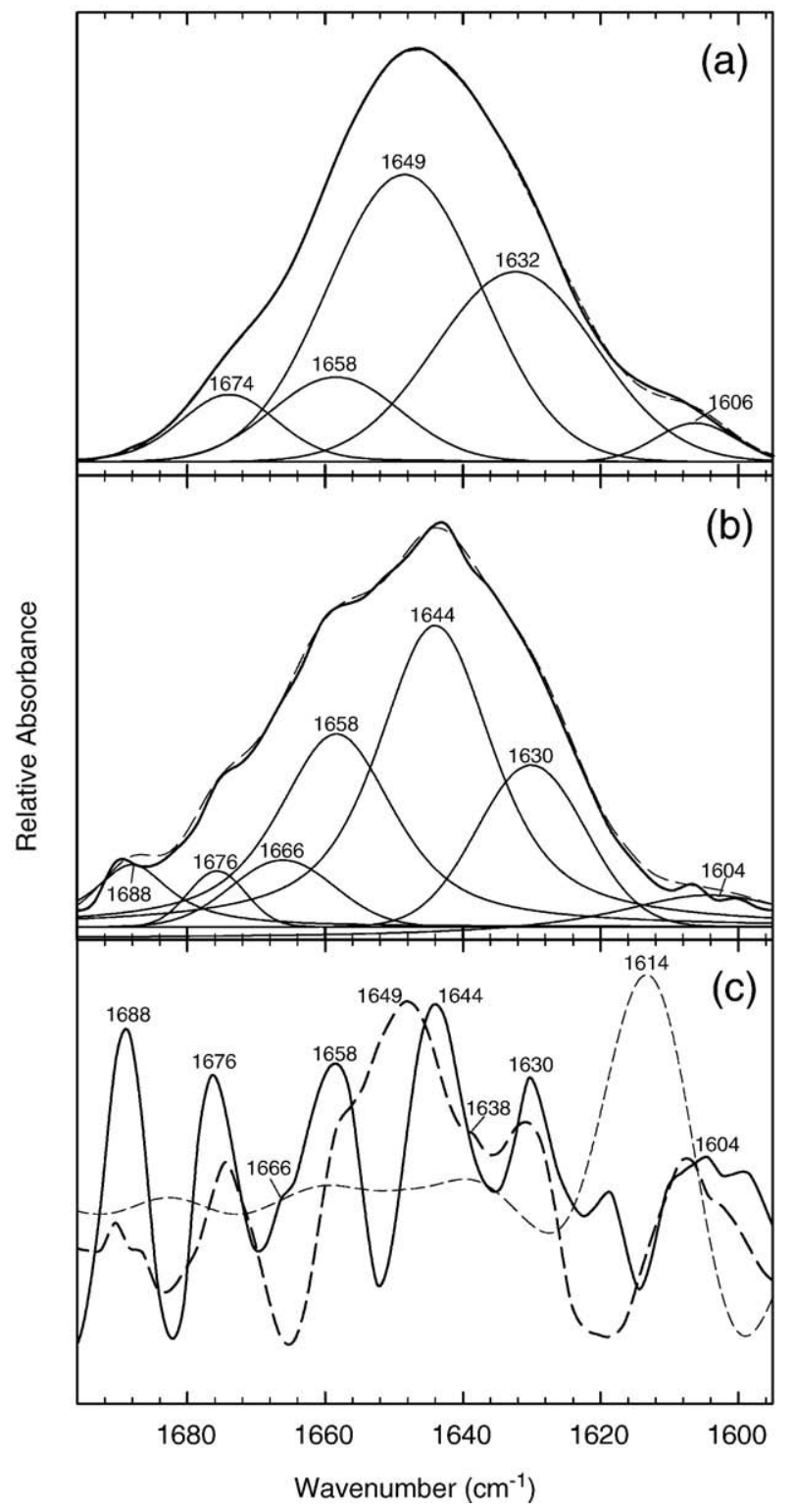

integrity of the protein (Fig. 2, bottom, inset). Monomeric human lysozyme prior to incubation (lane 1), a 15-day-incubated sample (lane 2), and an aliquot from an ultracentrifuged pellet of the latter (lane 4) and from its supernatant (lane 3) all migrate in a similar manner (Fig. 2, bottom, inset), indicating that no hydrolysis had occurred and that the protein molecules within the aggregates or present in solutions in which aggregation had occurred are all full-length lysozyme.

Lysozyme aggregation has also been followed by monitoring the degree of ANS binding, recording the increase in the fluorescence emission at $475 \mathrm{~nm}$ of ANS added to aliquots withdrawn from the aggregation mixture at different time points (Fig. 2, bottom, open circles). The experimental data points do not fit to a sigmoidal curve but show an initial increase during the previously detected lag phase, a sudden increase corresponding to the growth phase observed in the ThT fluorescence experiment, and then a plateau region at about 15 days. In order to distinguish whether the ANS fluorescence was due to the soluble oligomers or to the amyloid fibrils present in solution, we ultracentrifuged aliquots

Fig. 3. FTIR spectra of (a) monomeric lysozyme dissolved in $20 \mathrm{mM}$ glycine. $\mathrm{DCl}, \mathrm{pH}^{*} 3.0$ (uncorrected for isotopic effects) and (b) lysozyme oligomers produced after 5 days of incubation of the protein at $60^{\circ} \mathrm{C}$ in the same buffer. (c) The second derivatives of the amide I bands of lysozyme oligomers (continuous line) and of the monomeric protein (broken line) were used to identify the different spectral components. The thin broken line represents the second derivative of the spectrum of mature amyloid fibrils obtained previously at low $\mathrm{pH} .{ }^{14}$ Curve fitting was performed with Gaussian and Lorentzian lineshapes and with bandwidths varying between 15 and $20 \mathrm{~cm}^{-1} .42$ The heights, widths, and positions of each band were optimized iteratively. ${ }^{43,44}$ The sum of the fitted curves is shown as a broken line, closely overlapping the experimental trace, shown as a continuous line ( $a$ and $b$ ). Deuterated lysozyme was prepared by dissolving the protein in $\mathrm{D}_{2} \mathrm{O}$ at $\mathrm{pH}^{*} 3.8$ at a concentration of $0.68 \mathrm{mM}$, filtering the protein solution with a $20-\mathrm{nm}$ pore-size filter (Whatman) and incubating it at $80^{\circ} \mathrm{C}$ for $40 \mathrm{~min}$, followed by lyophilization. ${ }^{45}$ The spectrum in (a) was registered after dissolving the monomeric deuterated protein in $20 \mathrm{mM}$ glycine $\cdot \mathrm{DCl}, \mathrm{pH} * 3.0$, at a $5 \mathrm{mg} / \mathrm{ml}$ concentration. The spectrum in (b) was registered after separating lysozyme oligomers from the monomeric protein by filtration with Microcon YM-100 ultrafilters, following the procedure suggested by the supplier (Millipore, Bedford, MA, USA). The concentration of the purified oligomers collected on the filter was $1 \mathrm{mg} / \mathrm{ml}$, as evaluated by UV absorbance measurement. FTIR spectra were recorded at $20-22{ }^{\circ} \mathrm{C}$ using a Perkin Elmer 1720X spectrometer (Norwalk), purged with a continuous flow of $\mathrm{N}_{2}$ gas. Protein samples were placed between a pair of $\mathrm{CaF}_{2}$ windows separated by a $50-\mu \mathrm{m}$ Mylar spacer. For each protein sample, 50 interferograms were accumulated at a spectral resolution of $2 \mathrm{~cm}^{-1}$. The spectra were analyzed using the Grams 32 program version 4.14 (Galactic Industries Corporation, Salem, NH). Buffer spectra were recorded under conditions identical with those used for protein samples and subtracted from the spectra of the latter. 
Table 1. Secondary-structure content of monomeric and aggregated human lysozyme, as determined by FTIR (see Fig. 3)

\begin{tabular}{|c|c|c|c|c|}
\hline Wavenumber ${ }^{\mathrm{a}}\left(\mathrm{cm}^{-1}\right)$ & Structural assignment & Monomer $(\%)^{b}$ & Oligomers $(\%)^{\mathrm{b}}$ & Mature fibrils $(\%)^{\mathrm{b}}$ \\
\hline $1614-1630$ & Aggregated $\beta$-sheet & - & 17 & 32 \\
\hline 1632 & Antiparallel $\beta$-sheet & 32 & - & - \\
\hline $1641-1644$ & Disordered/Loops & - & 41 & 47 \\
\hline 1649 & $\alpha$-Helix & 49 & - & - \\
\hline 1658 & Turns & 12 & 27 & 14 \\
\hline 1666 & Turns & - & 7 & - \\
\hline 1674-1676 & Antiparallel $\beta$-sheet & 7 & 3 & - \\
\hline 1688 & Aggregated $\beta$-sheet & - & 5 & 7 \\
\hline
\end{tabular}

a Peak position of the amide I band components, as deduced by the second-derivative spectra.

b Percentage area of the amide I band components, as obtained by integrating the area under each deconvoluted band. The areas corresponding to side-chain contributions located at 1604-1606 cm $\mathrm{cm}^{-1}$ have not been considered.

removed from the reaction mixture at the different time points, and the ANS binding assay was repeated for the supernatants, where only protein monomers and soluble oligomers are present, thereby excluding any contribution to the fluorescence enhancement from fibrils (Fig. 2, bottom, filled circles). The fluorescence spectrum of ANS in the presence of the monomeric protein before incubation, collected at pH3.0 at $20{ }^{\circ} \mathrm{C}$, is identical with that in the absence of the protein, both in terms of maximum emission wavelength (510-516 nm) and intensity, indicating that monomeric lysozyme does not bind ANS, a result attributable to its existence in a native-like conformation ${ }^{29}$ (data not shown). By contrast, the fluorescence emission at $475 \mathrm{~nm}$ of ANS for the supernatants reveals that even after 2 days of incubation, a stable protein species capable of binding ANS is present and suggests that the quantity of this species increases slowly for up to 20 days of incubation. TEM pictures show that after 2 days of incubation, small quantities of spherical aggregates are present in the samples (not shown). This result indicates that the lysozyme oligomers visible by TEM must contain substantial regions of exposed hydrophobic surface and, therefore, suggests that a major conformational rearrangement has taken place in the protein during incubation. Amyloid fibrils formed after 12 days of incubation bind ANS, as already reported for other systems, indicating that they also possess nonnative surfaces. ${ }^{34,41}$ Since the quantum yield of ANS is likely to increase considerably with increasing amyloid aggregate size, the latter suggests that the binding of ANS to the oligomers could be much greater than the binding to the fibrils.

The secondary structure of lysozyme when it is in an oligomer was assessed by FTIR and compared to that of the protein before incubation (Fig. 3). The FTIR spectra have been deconvoluted into their component bands, each of which can be associated with specific elements of secondary structure. ${ }^{46,47}$ The FTIR spectrum of the monomeric protein at pH3.0 is indicative of the characteristic native fold, with a prevalence of $\alpha$-helical structure at $1649 \mathrm{~cm}^{-1}$ (49\%), of antiparallel $\beta$-sheet structure, suggested by the presence of the two characteristic bands at 1632 and $1674 \mathrm{~cm}^{-1}$ (39\%), and of turns giving rise to the component at $1658 \mathrm{~cm}^{-1}(12 \%)^{48,49}$ (Fig. 3a and
Table 1). The FTIR spectrum of lysozyme oligomers formed after 5 days of incubation at pH3.0 and isolated by ultrafiltration shows substantial changes when compared to that of the monomer, both in terms of the positions and intensities of the bands (Fig. $3 b$ and c). In particular, the main band is shifted to a lower wavenumber $\left(1644 \mathrm{~cm}^{-1}, 41 \%\right)$, indicating that some of the $\alpha$-helical structure present in the monomer has been lost as a result of a partial unfolding of the polypeptide chain. Moreover, two new bands at 1630 and $1688 \mathrm{~cm}^{-1}$ are evident, accounting for $22 \%$ of the structure, which are typical of the intermolecular $\beta$-sheet structure present in amyloid fibrils, as the absorption maxima at $\sim 1632$ and $1674 \mathrm{~cm}^{-1}$, characteristic of antiparallel $\beta$-sheet structure, shift to 1611-1630 and $\sim 1684 \mathrm{~cm}^{-1}$, respectively. ${ }^{50}$ Finally, the FTIR spectrum of the oligomers shows that the components at 1658 and $1666 \mathrm{~cm}^{-1}$ characteristic of turns are substantially increased (34\%).

It has been reported previously that the FTIR spectrum of mature lysozyme fibrils at low $\mathrm{pH}$ is characterized by a large contribution of the bands at 1614 and $1688 \mathrm{~cm}^{-1}$ (32\% and $7 \%$, respectively), corresponding to $\beta$-structure, but that the main contribution is still at $1641 \mathrm{~cm}^{-1}(47 \%)$, corresponding to random structure, plus a $14 \%$ content of turns indicated by a band at $1658 \mathrm{~cm}^{-1} .14$ Therefore, as the aggregation process of lysozyme proceeds, the amount of $\beta$-structure in the aggregates increases even though the entire polypeptide chain does not become involved in this type of structure. ${ }^{14}$

A proteolysis experiment with pepsin was also conducted on the soluble prefibrillar lysozyme aggregates and on monomeric lysozyme prior to incubation (Fig. 4). The aim was to gain information on the structure and dynamics of lysozyme in its monomeric and aggregated forms, since proteolytic cleavage generally occurs at flexible regions of a polypeptide chain that are devoid of hydrogenbonded regular secondary structure such as $\alpha$ helices and $\beta$-strands. ${ }^{52-54}$ The proteolysis reactions were conducted at $57^{\circ} \mathrm{C}$, after lowering the $\mathrm{pH}$ of the solution to $\mathrm{pH} 2.0$ with aqueous $\mathrm{HCl}$, since, at this $\mathrm{pH}$, pepsin exhibits its maximum activity. ${ }^{55}$ The first peptide bonds cleaved by pepsin in the polypeptide chain of monomeric lysozyme at pH2.0 are Phe57-Gln58 and Ala108-Trp109, located 
(a)

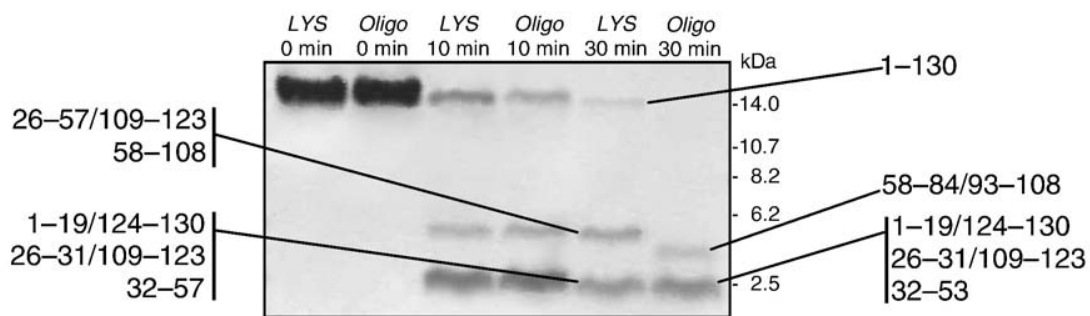

(b)

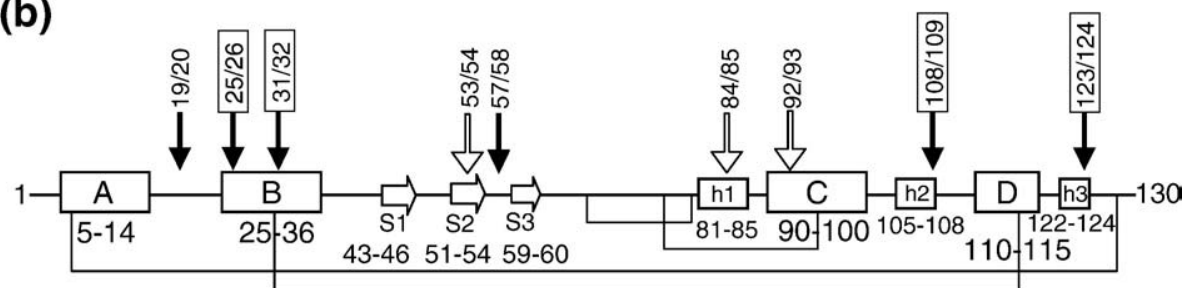

Fig. 4. Susceptibility to proteolysis with pepsin of wild-type human lysozyme in its monomeric, oligomeric, and fibrillar states. (a) SDS-PAGE analysis of the proteolysis with pepsin of an oligomer-containing sample formed after 5 days of incubation of lysozyme at pH3.0 and $60^{\circ} \mathrm{C}$ (Oligo) and of monomeric lysozyme (LYS). SDS-PAGE was conducted under nonreducing conditions. ${ }^{51}$ The main proteolytic fragments are indicated near the corresponding electrophoretic bands. Some fragments are nicked species linked by the disulfide bonds of the protein. The proteolysis reactions were conducted at $\mathrm{pH} 2.0$ at $57^{\circ} \mathrm{C}$ with enzyme:substrate $=1: 50$ (by weight) of pepsin. Aliquots from the proteolysis mixtures were withdrawn after 10 and $30 \mathrm{~min}$ of incubation, and the reactions were quenched with an equal volume of aqueous $\mathrm{NH}_{3}(1: 100, \mathrm{v} / \mathrm{v})$. The identity of the protein fragments separated by reverse-phase HPLC was assessed by electrospray ionization-mass spectrometry with a Q-Tof Micro mass spectrometer (Micromass, Manchester, UK). (b) Schematic representation of the secondary structure of human lysozyme ${ }^{48}$ indicating the proteolytic sites obtained after digestion with pepsin. The boxes indicate the location along the 130 -residue chain of the protein of $\alpha$-helices (A-D) and of the $3_{10}$ helical elements (h1-h3), while the arrows represent the three $\beta$-strands (S1-S3). The connectivity of the four disulfide bonds Cys6-Cys128, Cys30-Cys116, Cys65-Cys81, and Cys77-Cys95 of the protein is indicated by continuous lines. Black and white arrows indicate the pepsin cleavage sites on the monomeric protein and the additional sites of cleavages on the protein in the prefibrillar form, respectively. Boxed arrows indicate the sites of cleavage of lysozyme in the mature amyloid fibrils studied previously. ${ }^{14}$

in the native structure in the $\beta$-domain and in the $3_{10}$ helix between helices $C$ and $D$, respectively, followed by the peptide bonds Gly19-Tyr20, Leu25Ala26, Leu31-Ala32, and Gln123-Tyr124 (see Table S1). These results indicate that the regions of the sequence encompassing these peptide bonds are unfolded enough, for at least a significant fraction of time, to allow the attack of the protease molecule (Fig. $4 \mathrm{a}$ and b). Interestingly, the proteolysis of lysozyme in its oligomeric form leads to the formation of the same peptic fragments as it does in the monomeric, partially unfolded state. ${ }^{19,22}$ Remarkably, however, within the oligomers, lysozyme is digested to a greater extent if compared with the monomeric state; after $30 \mathrm{~min}$ of incubation, no intact protein is evident in the proteolytic mixture, and most importantly, the three peptide bonds Asp53-Tyr54, Leu84-Leu85, and Ala92Val93, which are located in the C-helix and the $\beta$ domain, are also cleaved (Fig. $4 \mathrm{a}$ and b), while for the monomeric protein, the degree of degradation at these peptide bonds is negligible. These results indicate that the polypeptide chain of lysozyme in the oligomeric state is more unstructured than that in the monomeric form, especially in the segments encompassing the $\beta$-domain and the C-helix of the native protein. In addition, it has previously been shown that mature amyloid fibrils produced at low
$\mathrm{pH}$ experience detectable proteolysis at very few sites in the sequence, despite the fact that there are many potential peptic sites distributed along the chain, indicating that the polypeptide chain of lysozyme embedded into mature fibrils is arranged in a largely compact and stable structure (Fig. 4c). ${ }^{14}$ Furthermore, proteolysis with pepsin of fully unfolded lysozyme, when the disulfide bonds of the protein are reduced and alkylated, conducted under the same conditions, leads to the formation of short peptides resulting from cleavage all along the sequence, indicating that if the protein chain is devoid of any fixed structure (as in this case), ${ }^{56}$ it is essentially fully accessible to the protease (data not shown).

\section{General insights into amyloid disease}

The existence of aggregates with toxic activity formed early on the aggregation pathway of lysozyme from various species has been shown by several groups. ${ }^{10-13,57}$ The aim of the present study was to obtain detailed structural information about the early aggregates formed by wild-type human lysozyme at acidic $\mathrm{pH}$ and moderately high temperature. During the aggregation process, lysozyme forms spherical oligomers that, upon prolonged incubation, convert into mature amyloid 
fibrils. Much evidence suggests that the former species could be those with high toxicity. An important finding of this study is the low concentration in the solution of oligomeric species $(\sim 5 \%)$ under the conditions used here. As it was recently reported that the extracellular chaperone clusterin is able to inhibit the aggregation of the amyloidogenic I56T variant of lysozyme, an observation attributed to its specific interaction with oligomeric species formed at low population (less than 5\%) during the lag phase of the aggregation process; ${ }^{57,58}$ species of this type are likely to be fundamental for triggering the aggregation of lysozyme even if present only in low concentration.

Another remarkable characteristic of the lysozyme oligomers reported here is the hydrophobicity of their surfaces, as revealed by their avid binding of ANS. The exposure of hydrophobic regions able to bind ANS is also associated with the formation of soluble early aggregates of lysozyme at $\mathrm{pH} 12.2 .^{59}$ ANS has similarly been reported to interact with oligomeric aggregates of other proteins; ${ }^{34}$ for example, well before the formation of amyloid fibrils able to bind ThT, insulin begins to undergo a series of structural changes, detected by binding with ANS. ${ }^{41}$ Crucially, regions of exposed hydrophobicity have been strongly implicated in the cytotoxicity of protein aggregates, as it has been proposed that the pathogenic nature of prefibrillar aggregates could lie in the exposure of groups that are normally buried in a folded protein. 1,3,60 Interestingly, the ANS signal arising from oligomeric species of lysozyme does not decrease over time, it even slightly increases, despite the fact that the concentration of soluble protein is progressively reduced and that the equilibrium is shifted toward aggregated species. Indeed, ultrafiltration analysis indicates that, after 5 days, oligomers involve only $5 \%$ of lysozyme molecules in solution, while the rest are in the monomeric form, yet the overall ANS signal corresponds to $20 \%$ of its maximum value. When the aggregation process reaches a plateau (15 days), the ANS signal arising from oligomers (see Fig. 2, bottom, filled circles) is increased to $30 \%$ of the maximum value. Since ultracentrifugation experiments show that, at this time, $\sim 90 \%$ of the protein molecules are embedded into fibrils, the latter appear to exist in equilibrium with $\sim 7.5 \%$ of oligomeric species and $\sim 2.5 \%$ of monomers.

Lysozyme oligomers are digested by pepsin to a greater extent than is the monomeric protein, suggesting that lysozyme molecules within these species are more flexible and unfolded. This result is similar to the findings of a study of the SH3 domain of phosphoinositol-3-kinase, where soluble amyloid precursors formed at low $\mathrm{pH}$ were shown to be completely degraded by pepsin, although mature amyloid fibrils remain largely intact under the same conditions. ${ }^{61,62}$ Moreover, the observation that species formed early in the aggregation process of the fragment 105-115 of transthyretin are unstable under high hydrostatic pressure because they still contain cavities and packing defects suggests again that they are not yet highly ordered. ${ }^{63}$ In addition, the proteolysis data for lysozyme are in accord with the FTIR data reported here, which show that the initial aggregates possess a high content of random structure $(\sim 40 \%)$ but, at the same time, have some intermolecular $\beta$-structure $(22 \%)$. The presence of a significant fraction of random structure has also been observed by CD measurements in oligomers formed by the amyloid $\beta$ peptide. ${ }^{64}$ It therefore appears likely that these are common features of the oligomeric species formed by different peptides and proteins.

The current model proposed for lysozyme amyloid aggregation suggests that intermolecular interactions between those regions that are unfolded in the amyloidogenic intermediate (i.e., the C-helix and the $\beta$-domain) initiate the process that leads to the formation of mature amyloid fibrils. ${ }^{15,16}$ The data described here indicate that the formation of lysozyme oligomers also involves the unfolding of a substantial part of the remainder of the polypeptide chain, at least transiently, since the oligomers are more unstructured and sensitive to proteolysis, and also expose nonnative cluster of hydrophobic groups on their surfaces. Moreover, FTIR data suggest that at least part of this unfolding process results in the formation of an initial stretch of intermolecular $\beta$-structure, an event that is likely to promote nonnative interactions elsewhere in the molecule and, thus, to help stabilize oligomeric assemblies. Indeed, if the entire region suggested to be unfolded, corresponding to $45-58 \%$ of the lysozyme sequence, ${ }^{14-16}$ was already embedded in the cross- $\beta$ core of the oligomer, the $\beta$-sheet content of the oligomers would be greater than the $22 \%$ found from the FTIR analysis.

As aggregation proceeds, the lysozyme chain converts progressively into $\beta$-structure; FTIR suggests that the proportion of $\beta$-sheet structure increases from $22 \%$ in prefibrillar aggregates to $39 \%$ in mature fibrils (see Table 1). The structural transitions that lead to increasing order in amyloid proteins have been compared to crystallization phenomena, and indeed, when polymers approach crystallinity, they have been observed to pass through species of increasing order on the way to their stable end products. ${ }^{65}$ In the case of lysozyme, it has previously been demonstrated, by means of proteolysis experiments, that in mature fibrils, both the $\beta$-domain and the $C$-helix of the native state are reorganized prior to incorporation within the $\beta$-core of the fibrils. ${ }^{14}$ The fact that lysozyme oligomers are completely and rapidly digested by pepsin, while mature fibrils are only partially and slowly digested, indicates that the species formed first during aggregation are likely to have the flexibility required for such a process to take place.

Overall, the results presented here shed light on the elusive conformational features of oligomeric species formed during the first phases of the aggregation process that results in peptides and proteins converting into amyloid fibrils. In the case of the pathogenic behavior of mutational variants of lysozyme, the aggregation process is enhanced 
relative to the wild-type protein as a result of the destabilization of the native state. ${ }^{21}$ In this systemic disease, the resulting proliferation of fibrils is undoubtedly the reason that large quantities of deposits are formed, which cause physical damage to organs such as the liver. ${ }^{1,66}$ The toxicity of the oligomers is, therefore, not necessarily the primary origin of the disease, although it may well play a very significant role. In many other cases, however, the oligomeric intermediates formed during fibril formation are highly likely to be the primary toxic agents involved in amyloid disease; this situation is, for example, probable in both type II diabetes and neurodegenerative diseases, where cell damage and cell death are clearly evident. The results of the present study could have general significance not only because they reveal the possible generic toxicity of misfolded protein oligomers but also because they are a key factor in defining our ability to rise to the challenge of developing therapeutic agents to prevent or combat these debilitating and increasingly prevalent conditions. As these conditions range from systemic deposition disorders to neurodegenerative disorders such as Alzheimer's disease and type II diabetes, this goal is particularly urgent because of the increasingly dominant role these diseases now play in the modern world.

\section{Acknowledgements}

We gratefully acknowledge the support from the Italian Ministry of University and Research (Projects PRIN-2006 and FIRB-2003 No. RBNEOPX83 on Protein Folding and Aggregation) and from the UK Biotechnology and Biological Sciences Research. We are likewise grateful for the program grants awarded by both the Leverhulme and Wellcome Trusts and for the funds from the European Commission (Project LSHM-CT-2006-037525). The excellent technical assistance of Marcello Zambonin and Giuseppe Tognon is also gratefully acknowledged.

\section{Supplementary Data}

Supplementary data associated with this article can be found, in the online version, at doi:10.1016/j. jmb.2009.01.049

\section{References}

1. Chiti, F. \& Dobson, C. M. (2006). Protein misfolding, functional amyloid, and human disease. Annu. Rev. Biochem. 75, 333-366.

2. Lansbury, P. T. \& Lashuel, H. A. (2006). A century-old debate on protein aggregation and neurodegeneration enters the clinic. Nature, 443, 774-779.

3. Bucciantini, M., Giannoni, E., Chiti, F., Baroni, F., Formigli, L., Zurdo, J. et al. (2002). Inherent toxicity of aggregates implies a common mechanism for protein misfolding diseases. Nature, 416, 507-511.
4. Stefani, M. \& Dobson, C. M. (2003). Protein aggregation and aggregate toxicity: new insights into protein folding, misfolding diseases and biological evolution. J. Mol. Med. 81, 678-699.

5. Kodali, R. \& Wetzel, R. (2007). Polymorphism in the intermediates and products of amyloid assembly. Curr. Opin. Struct. Biol. 17, 48-57.

6. Pepys, M. B., Hawkins, P. N., Booth, D. R., Vigushin, D. M., Tennent, G. A., Soutar, A. K. et al. (1993). Human lysozyme gene mutations cause hereditary systemic amyloidosis. Nature, 362, 553-557.

7. Valleix, S., Drunat, S., Philit, J. B., Adoue, D., Piette, J. C., Droz, D. et al. (2002). Hereditary renal amyloidosis caused by a new variant lysozyme W64R in a French family. Kidney Int. 61, 907-912.

8. Yazaki, M., Farrell, S. A. \& Benson, M. D. (2003). A novel lysozyme mutation Phe57Ile associated with hereditary renal amyloidosis. Kidney Int. 63, 1652-1657.

9. Röcken, C., Becker, K., Fändrich, M., Schroeckh, V., Stix, B., Rath, T. et al. (2006). A Lys amyloidosis caused by compound heterozygosity in exon 2 (Thr70Asn) and exon 4 (Trp112Arg) of the lysozyme gene. Hum. Mutat. 27, 119-120.

10. Demuro, A., Mina, E., Kayed, R., Milton, S. C., Parker, I. \& Glabe, C. G. (2005). Calcium dysregulation and membrane disruption as a ubiquitous neurotoxic mechanism of soluble amyloid oligomers. J. Biol. Chem. 280, 17294-17300.

11. Malisauskas, M., Ostman, J., Darinskas, A., Zamotin, V., Liutkevicius, E., Lundgren, E. \& Morozova-Roche, L. A. (2005). Does the cytotoxic effect of transient amyloid oligomers from common equine lysozyme in vitro imply innate amyloid toxicity? J. Biol. Chem. 280, 6269-6275.

12. Gharibyan, A. L., Zamotin, V., Yanamandra, K., Moskaleva, O. S., Margulis, B. A., Kostanyan, I. A. \& Morozova-Roche, L. A. (2007). Lysozyme amyloid oligomers and fibrils induce cellular death via different apoptotic/necrotic pathways. J. Mol. Biol. $365,1337-1349$.

13. Vieira, M. N., Forny-Germano, L., Saraiva, L. M., Sebollela, A., Martinez, A. M., Houzel, J. C. et al. (2007). Soluble oligomers from a non-disease related protein mimic Abeta-induced tau hyperphosphorylation and neurodegeneration. J. Neurochem. 103, 736-748.

14. Frare, E., Mossuto, M. F., Polverino de Laureto, P., Dumoulin, M., Dobson, C. M. \& Fontana, A. (2006). Identification of the core structure of lysozyme amyloid fibrils by proteolysis. J. Mol. Biol. 361, 551-561.

15. Canet, D., Last, A. M., Tito, P., Sunde, M., Spencer, A., Archer, D. B. et al. (2002). Local cooperativity in the unfolding of an amyloidogenic variant of human lysozyme. Nat. Struct. Biol. 9, 308-315.

16. Dumoulin, M., Canet, D., Last, A. M., Pardon, E., Archer, D. B., Muyldermans, S. et al. (2005). Reduced global cooperativity is a common feature underlying the amyloidogenicity of pathogenic lysozyme mutations. J. Mol. Biol. 25, 773-788.

17. Krebs, M. R., Wilkins, D. K., Chung, E. W., Pitkeathly, M. C., Chamberlain, A. K., Zurdo, J. et al. (2000). Formation and seeding of amyloid fibrils from wildtype hen lysozyme and a peptide fragment from the beta-domain. J. Mol. Biol. 14, 541-549.

18. Frare, E., Polverino de Laureto, P., Zurdo, J., Dobson, C. M. \& Fontana, A. (2004). A highly amyloidogenic region of hen lysozyme. J. Mol. Biol. 23, 1153-1165.

19. Haezebrouck, P., Joniau, M., Van Dael, H., Hooke, S. D., Woodruff, N. D. \& Dobson, C. M. (1995). An 
equilibrium partially folded state of human lysozyme at low pH. J. Mol. Biol. 246, 382-387.

20. Funahashi, J., Takano, K., Ogasahara, K., Yamagata, Y. \& Yutani, K. (1996). The structure, stability, and folding process of amyloidogenic mutant human lysozyme. J. Biochem. 120, 1216-1223.

21. Booth, D. R., Sunde, M., Bellotti, V., Robinson, C. V. Hutchinson, W. L., Fraser, P. E. et al. (1997). Instability, unfolding and aggregation of human lysozyme variants underlying amyloid fibrillogenesis. Nature, 27, 787-793.

22. Morozova-Roche, L. A., Zurdo, J., Spencer, A., Noppe, W., Receveur, V., Archer, D. B. et al. (2000). Amyloid fibril formation and seeding by wild-type human lysozyme and its disease-related mutational variants. J. Struct. Biol. 130, 339-351.

23. Goda, S., Takano, K., Yamagata, Y., Maki, S., Namba, K. \& Yutani, K. (2002). Elongation in a beta-structure promotes amyloid-like fibril formation of human lysozyme. J. Biochem. 132, 655-661.

24. Dumoulin, M., Kumita, J. R. \& Dobson, C. M. (2006). Normal and aberrant biological self-assembly: insights from studies of human lysozyme and its amyloidogenic variants. Acc. Chem. Res. 39, 603-610.

25. Dumoulin, M., Johnson, R. J. K., Bellotti, V. \& Dobson, C. M. (2007). Human lysozyme amyloidosis. In Protein Misfolding, Aggregation and Conformational Diseases. II. Molecular Basis of Conformational Diseases (Uversky, V. N. \& Fink, A. L., eds), pp. 285-308, Kluwer Academic/ Plenum Publishers, Dordrecht, The Netherlands.

26. Canfield, R. E., Kamerman, S., Sobel, H. H. \& Morgan, F. J. (1971). Primary structure of lysozymes from man and goose. Nat. New Biol. 232, 16-17.

27. Gill, S. G. \& von Hippel, P. H. (1989). Calculation of protein extinction coefficients from amino acid sequence data. Anal. Biochem. 182, 319-326.

28. Weber, G. \& Young, L. B. (1964). Fragmentation of bovine serum albumin by pepsin. I. The origin of the acid expansion of the albumin molecule. J. Biol. Chem. 239, 1415-1423.

29. Semisotnov, G. V., Rodionova, N. A., Razgulyaev, O. I., Uversky, V. N., Gripas', A. F. \& Gilmanshin, R. I. (1991). Study of the "molten globule" intermediate state in protein folding by a hydrophobic fluorescent probe. Biopolymers, 31, 119-128.

30. Li, A., Sowder, R. C., Henderson, L. E., Moore, S. P. Garfinkel, D. J. \& Fisher, R. J. (2001). Chemical cleavage at aspartyl residues for protein identification. Anal. Chem. 15, 5395-5402.

31. Meersman, F. \& Dobson, C. M. (2006). Probing the pressure-temperature stability of amyloid fibrils provides new insights into their molecular properties. Biochim. Biophys. Acta, 1764, 452-460.

32. Hoshi, M., Sato, M., Matsumoto, S., Noguchi, A., Yasutake, K., Yoshida, N. \& Sato, K. (2003). Spherical aggregates of beta-amyloid (amylospheroid) show high neurotoxicity and activate tau protein kinase I/ glycogen synthase kinase-3beta. Proc. Natl Acad. Sci. USA, 100, 6370-6375

33. Nandi, P. K. \& Nicole, J. C. (2004). Nucleic acid and prion protein interaction produces spherical amyloids which can function in vivo as coats of spongiform encephalopathy agent. J. Mol. Biol. 344, 827-837.

34. Lindgren, M., Sörgjerd, K. \& Hammarström, P. (2005). Detection and characterization of aggregates, prefibrillar amyloidogenic oligomers, and protofibrils using fluorescence spectroscopy. Biophys. J. 88, 4200-4212.

35. Steinhaus, H. (1999). Mathematical Snapshots. pp. 202-203, 3rd edit. Dover, Mineola, NY.
36. Orte, A., Birkett, N. R., Clarke, R. W., Devlin, G. L., Dobson, C. M. \& Klenerman, D. (2008). Direct characterization of amyloidogenic oligomers by singlemolecule fluorescence. Proc. Natl Acad. Sci. USA, 105, 14424-14429.

37. Kahle, P. J., Neumann, M., Ozmen, L., Müller, V., Odoy, S., Okamoto, N. et al. (2001). Selective insolubility of alpha-synuclein in human Lewy body diseases is recapitulated in a transgenic mouse model. Am. J. Pathol. 159, 2215-2225.

38. Walsh, D. M., Klyubin, I., Fadeeva, J. V., Cullen, W. K., Anwyl, R., Wolfe, M. S. et al. (2002). Naturally secreted oligomers of amyloid beta protein potently inhibit hippocampal long-term potentiation in vivo. Nature, 416, 535-539.

39. Sharon, R., Bar-Joseph, I., Frosch, M. P., Walsh, D. M., Hamilton, J. A. \& Selkoe, D. J. (2003). The formation of highly soluble oligomers of alpha-synuclein is regulated by fatty acids and enhanced in Parkinson's disease. Neuron, 37, 583-595.

40. LeVine, H. (1993). Thioflavine $\mathrm{T}$ interaction with synthetic Alzheimer's disease beta-amyloid peptides: detection of amyloid aggregation in solution. Protein Sci. 2, 404-410.

41. Ahmad, A., Uversky, V. N., Hong, D. \& Fink, A. L. (2005). Early events in the fibrillation of monomeric insulin. J. Biol. Chem. 280, 42669-42675.

42. Byler, D. M. \& Susi, H. (1986). Examination of the secondary structure of proteins by deconvolved FTIR spectra. Biopolymers, 25, 469-487.

43. Arrondo, J. L., Castresana, J., Valpuesta, J. M. \& Goni, F. M. (1994). Structure and thermal denaturation of crystalline and noncrystalline cytochrome oxidase as studied by infrared spectroscopy. Biochemistry, 33, 11650-11655.

44. García-García, J., Corbalan-Garcia, S. \& GomezFernandez, J. C. (1999). Effect of calcium and phosphatidic acid binding on the C2 domain of PKC alpha as studied by Fourier transform infrared spectroscopy. Biochemistry, 38, 9667-9675.

45. Chung, E. W., Nettleton, E. J., Morgan, C. J., Gross, M., Miranker, A., Radford, S. E. et al. (1997). Hydrogen exchange properties of proteins in native and denatured states monitored by mass spectrometry and NMR. Protein Sci. 6, 1316-1324.

46. Krimm, S. \& Bandekar, J. (1986). Vibrational spectroscopy and conformation of peptides, polypeptides and proteins. Adv. Protein Chem. 38, 181-364.

47. Surewicz, W. K. \& Mantsch, H. H. (1988). New insight into protein secondary structure from resolution-enhanced infrared spectra. Biochim. Biophys. Acta, 952, 115-130.

48. Blake, C. C., Pulford, W. C. \& Artymiuk, P. J. (1983). Xray studies of water in crystals of lysozyme. J. Mol. Biol. 167, 693-723.

49. Redfield, C. \& Dobson, C. M. (1990). ${ }^{1} \mathrm{H}$ NMR studies of human lysozyme: spectral assignment and comparison with hen lysozyme. Biochemistry, 29, 7201-7214.

50. Zandomeneghi, G., Krebs, M. R., McCammon, M. G. \& Fändrich, M. (2004). FTIR reveals structural differences between native beta-sheet proteins and amyloid fibrils. Protein Sci. 13, 3314-3321.

51. Schägger, H. \& Von Jagow, G. (1987). Tricine sodium dodecyl sulfate polyacrylamide gel electrophoresis for the separation of proteins in the range from $1 \mathrm{kDa}$ to 100 kDa. Anal. Biochem. 166, 368-379.

52. Fontana, A., Polverino de Laureto, P., De Filippis, V., Scaramella, E. \& Zambonin, M. (1997). Probing the partly folded states of proteins by limited proteolysis. Folding Des. 2, R17-R26. 
53. Hubbard, S. J. (1998). The structural aspects of limited proteolysis of native proteins. Biochim. Biophys. Acta 1382, 191-206.

54. Fontana, A., Polverino de Laureto, P., Spolaore, B., Frare, E., Picotti, P. \& Zambonin, M. (2004). Probing protein structure by limited proteolysis. Acta Biochim. Pol. 51, 299-321.

55. Fruton, J. S. (1970). The specificity and mechanism of pepsin action. Adv. Enzymol. Relat. Areas Mol. Biol. 33 401-443.

56. Schwalbe, H., Fiebig, K. M., Buck, M., Jones, J. A., Grimshaw, S. B., Spencer, A. et al. (1997). Structural and dynamical properties of a denatured protein. Heteronuclear 3D NMR experiments and theoretical simulations of lysozyme in $8 \mathrm{M}$ urea. Biochemistry, $\mathbf{3 6}$ 8977-8991.

57. Yerbury, J. J., Poon, S., Meehan, S., Thompson, B., Kumita, J. R., Dobson, C. M. \& Wilson, M. R. (2007). The extracellular chaperone clusterin influences amyloid formation and toxicity by interacting with prefibrillar structures. FASEB J. 21, 2312-2322.

58. Kumita, J. R., Poon, S., Caddy, G. L., Hagan, C. L., Dumoulin, M., Yerbury, J. J. et al. (2007). The extracellular chaperone clusterin potently inhibits human lysozyme amyloid formation by interacting with prefibrillar species. J. Mol. Biol. 369, 157-167.

59. Kumar, S., Ravi, V. K. \& Swaminathan, R. (2008). How do surfactants and DTT, affect the size, dynamics, activity and growth of soluble lysozyme aggregates? Biochem. J. 415, 275-288.

60. Cheon, M., Chang, I., Mohanty, S., Luheshi, L. M., Dobson, C. M., Vendruscolo, M. \& Favrin, G. (2007). Structural reorganisation and potential toxicity of oligomeric species formed during the assembly of amyloid fibrils. PLoS Comput. Biol. 3, 1727-1738.

61. Zurdo, J., Guijarro, J. I. \& Dobson, C. M. (2001). Preparation and characterization of purified amyloid fibrils. J. Am. Chem. Soc. 123, 8141-8142.

62. Polverino de Laureto, P., Taddei, N., Frare, E., Capanni, C., Costantini, S., Zurdo, J. et al. (2003). Protein aggregation and amyloid fibril formation by an SH3 domain probed by limited proteolysis. J. Mol. Biol. 334, 129-141.

63. Dirix, C., Meersman, F., MacPhee, C. E., Dobson, C. M. \& Heremans, K. (2005). High hydrostatic pressure dissociates early aggregates of TTR105-115, but not the mature amyloid fibrils. J. Mol. Biol. 347, 903-909.

64. Bitan, G., Kirkitadze, M. D., Lomakin, A., Vollers, S. S., Benedek, G. B. \& Teplow, D. B. (2003). Amyloid betaprotein (Abeta) assembly: Abeta 40 and Abeta 42 oligomerize through distinct pathways. Proc. Natl Acad. Sci. USA, 100, 330-335.

65. Elias, H.-G. (2003). An Introduction to Plastics, 2nd edit. Wiley-VCH, Weinheim, Germany.

66. Pepys, M. B. (2006). Amyloidosis. Annu. Rev. Med. 57, 223-241. 